\title{
Correction to $\alpha$-Glucosidase Inhibitors from Ageratina grandifolia
}

José Alberto Gutiérrez-González, Araceli Pérez-Vásquez, Rafael Torres-Colín, Manuel Rangel-Grimaldo, Daniela Rebollar-Ramos, and Rachel Mata*

J. Nat. Prod. 2021, 84, 1573-1578, doi.org/10.1021/acs.jnatprod.1c00105

Table S1. Shielding Tensors and NMR Single-Point Calculations at DFT B3LYP/6-311G+(2d,p)//M062X/6$31+g(d, p)$ Level Of Theory for the Proposed 2,2-Dimethyl-3-Hidroxy-4-(1-Angeloyloxy)Chromane Structure.

Table S2. Calculated Coupling Constants (Fermi Contacts) at DFT B3LYP/6-311G+(2d,p)//M062X/6-31+g(d,p) Level of Theory for the Proposed 2,2-Dimethyl-3-Hidroxy-4-(1-Angeloyloxy)Chromane Structure.

Table S3. Geometry Optimization and Boltzmann Distribution at M062X/6-31+g(d,p) Level of Theory for the Diastereomers of the Proposed 2,2-Dimethyl-3-Hidroxy-4-(1-Angeloyloxy)Chromane Structure.

Table S4. DP4+ Analysis of the Proposed 2,2-Dimethyl-3-Hidroxy-4-(1-Angeloyloxy)Chromane Structure.

Table S5 J-DP4 Analysis of the Proposed 2,2-Dimethyl-3-Hidroxy-4-(1-Angeloyloxy)Chromane Structure. 
Table S1. Shielding Tensors and NMR Single-Point Calculations at DFT M062X/6-31+g(d,p) Level Of

Theory for the Proposed 2,2-Dimethyl-3-Hidroxy-4-(1-Angeloyloxy)Chromane Structure.

\begin{tabular}{|c|c|c|c|c|c|c|c|c|c|c|c|c|c|}
\hline \multirow[b]{2}{*}{ Pos } & \multirow[b]{2}{*}{$3 R, 4 R$} & \multicolumn{2}{|c|}{$\begin{array}{l}\text { Isotropic Tensors } \\
\qquad\left({ }^{13} \mathrm{C}\right)\end{array}$} & \multirow[b]{2}{*}{$3 S, 4 S$} & \multicolumn{4}{|c|}{$\begin{array}{l}\text { Calculated Chemical } \\
\text { Shifts }\left({ }^{13} \mathrm{C}\right)^{a}\end{array}$} & \multirow[b]{2}{*}{ Exp. } & \multicolumn{4}{|c|}{$\left|\delta_{\text {teo }}-\delta_{\text {exp }}\right|$} \\
\hline & & $3 S, 4 R$ & $3 R, 4 S$ & & $3 R, 4 R$ & $3 S, 4 R$ & $3 R, 4 S$ & $3 S, 4 S$ & & $3 R, 4 R$ & $3 S, 4 R$ & $3 R, 4 S$ & $3 S, 4 S$ \\
\hline 2 & $\begin{array}{c}2.57203 \\
5\end{array}$ & $\begin{array}{c}3.18753 \\
2\end{array}$ & $\begin{array}{c}0.69311 \\
2\end{array}$ & $\begin{array}{c}0.69531 \\
5\end{array}$ & $\begin{array}{c}77.0579 \\
7\end{array}$ & $\begin{array}{c}76.4424 \\
7\end{array}$ & $\begin{array}{c}78.9368 \\
9\end{array}$ & $\begin{array}{c}78.9346 \\
9\end{array}$ & 79.63 & $\begin{array}{c}2.57203 \\
5\end{array}$ & $\begin{array}{c}3.18753 \\
2\end{array}$ & $\begin{array}{c}0.69311 \\
2\end{array}$ & $\begin{array}{c}0.69531 \\
5\end{array}$ \\
\hline 3 & $\begin{array}{c}1.10682 \\
3\end{array}$ & $\begin{array}{c}3.33535 \\
7\end{array}$ & $\begin{array}{c}0.64771 \\
1\end{array}$ & $\begin{array}{c}8.74772 \\
1\end{array}$ & $\begin{array}{c}73.0831 \\
8\end{array}$ & $\begin{array}{c}70.8546 \\
4\end{array}$ & $\begin{array}{c}73.5422 \\
9\end{array}$ & $\begin{array}{c}65.4422 \\
8\end{array}$ & 74.19 & $\begin{array}{c}1.10682 \\
3\end{array}$ & $\begin{array}{c}3.33535 \\
7\end{array}$ & $\begin{array}{c}0.64771 \\
1\end{array}$ & $\begin{array}{c}8.74772 \\
1\end{array}$ \\
\hline 4 & $\begin{array}{c}5.95922 \\
8\end{array}$ & $\begin{array}{c}4.07800 \\
4\end{array}$ & $\begin{array}{c}0.04442 \\
1\end{array}$ & $\begin{array}{c}4.32388 \\
9\end{array}$ & $\begin{array}{c}65.9007 \\
7\end{array}$ & 67.782 & $\begin{array}{c}71.8155 \\
8\end{array}$ & $\begin{array}{c}67.5361 \\
1\end{array}$ & 71.86 & $\begin{array}{c}5.95922 \\
8\end{array}$ & $\begin{array}{c}4.07800 \\
4\end{array}$ & $\begin{array}{c}0.04442 \\
1\end{array}$ & $\begin{array}{c}4.32388 \\
9\end{array}$ \\
\hline $4 a$ & $\begin{array}{c}0.20830 \\
2\end{array}$ & $\begin{array}{c}0.96694 \\
4\end{array}$ & $\begin{array}{c}0.18348 \\
6\end{array}$ & 2.39822 & $\begin{array}{c}119.321 \\
7\end{array}$ & $\begin{array}{c}118.563 \\
1\end{array}$ & $\begin{array}{c}119.346 \\
5\end{array}$ & $\begin{array}{c}117.131 \\
8\end{array}$ & $\begin{array}{c}119.5 \\
3\end{array}$ & $\begin{array}{c}0.20830 \\
2\end{array}$ & $\begin{array}{c}0.96694 \\
4\end{array}$ & $\begin{array}{c}0.18348 \\
6\end{array}$ & 2.39822 \\
\hline 5 & $\begin{array}{c}1.40803 \\
2\end{array}$ & $\begin{array}{c}3.25072 \\
1\end{array}$ & 1.51144 & $\begin{array}{c}1.44039 \\
2\end{array}$ & 131.128 & $\begin{array}{c}132.970 \\
7\end{array}$ & $\begin{array}{c}128.208 \\
6\end{array}$ & $\begin{array}{c}128.279 \\
6\end{array}$ & $\begin{array}{c}129.7 \\
2\end{array}$ & $\begin{array}{c}1.40803 \\
2\end{array}$ & $\begin{array}{c}3.25072 \\
1\end{array}$ & 1.51144 & $\begin{array}{c}1.44039 \\
2\end{array}$ \\
\hline 6 & $\begin{array}{c}3.34915 \\
8\end{array}$ & $\begin{array}{c}2.97689 \\
9\end{array}$ & 3.73521 & $\begin{array}{c}3.16803 \\
1\end{array}$ & $\begin{array}{c}127.050 \\
8\end{array}$ & $\begin{array}{c}127.423 \\
1\end{array}$ & $\begin{array}{c}126.664 \\
8\end{array}$ & 127.232 & 130.4 & $\begin{array}{c}3.34915 \\
8\end{array}$ & $\begin{array}{c}2.97689 \\
9\end{array}$ & 3.73521 & $\begin{array}{c}3.16803 \\
1\end{array}$ \\
\hline 7 & $\begin{array}{c}1.30075 \\
4\end{array}$ & $\begin{array}{c}2.17057 \\
7\end{array}$ & $\begin{array}{c}2.47763 \\
4\end{array}$ & $\begin{array}{c}2.32550 \\
7\end{array}$ & $\begin{array}{c}129.099 \\
2\end{array}$ & $\begin{array}{c}128.229 \\
4\end{array}$ & $\begin{array}{c}127.922 \\
4\end{array}$ & $\begin{array}{c}128.074 \\
5\end{array}$ & 130.4 & $\begin{array}{c}1.30075 \\
4\end{array}$ & $\begin{array}{c}2.17057 \\
7\end{array}$ & $\begin{array}{c}2.47763 \\
4\end{array}$ & $\begin{array}{c}2.32550 \\
7\end{array}$ \\
\hline 8 & $\begin{array}{c}2.26208 \\
2\end{array}$ & $\begin{array}{c}2.37503 \\
1\end{array}$ & $\begin{array}{c}3.03175 \\
1\end{array}$ & $\begin{array}{c}2.85500 \\
3\end{array}$ & $\begin{array}{c}115.197 \\
9\end{array}$ & 115.085 & $\begin{array}{c}114.428 \\
2\end{array}$ & 114.605 & $\begin{array}{c}117.4 \\
6\end{array}$ & $\begin{array}{c}2.26208 \\
2\end{array}$ & $\begin{array}{c}2.37503 \\
1\end{array}$ & $\begin{array}{c}3.03175 \\
1\end{array}$ & $\begin{array}{c}2.85500 \\
3\end{array}$ \\
\hline $8 a$ & $\begin{array}{c}1.07762 \\
6\end{array}$ & 1.64115 & $\begin{array}{c}1.74747 \\
3\end{array}$ & $\begin{array}{c}1.95922 \\
9\end{array}$ & $\begin{array}{c}156.122 \\
4\end{array}$ & $\begin{array}{c}155.558 \\
8\end{array}$ & $\begin{array}{c}155.452 \\
5\end{array}$ & $\begin{array}{c}155.240 \\
8\end{array}$ & 157.2 & $\begin{array}{c}1.07762 \\
6\end{array}$ & 1.64115 & $\begin{array}{c}1.74747 \\
3\end{array}$ & $\begin{array}{c}1.95922 \\
9\end{array}$ \\
\hline 9 & $\begin{array}{c}1.95979 \\
1\end{array}$ & $\begin{array}{c}2.18610 \\
8\end{array}$ & $\begin{array}{c}3.99401 \\
6\end{array}$ & $\begin{array}{c}1.00071 \\
5\end{array}$ & $\begin{array}{c}17.6802 \\
1\end{array}$ & $\begin{array}{c}21.8261 \\
1\end{array}$ & $\begin{array}{c}15.6459 \\
8\end{array}$ & $\begin{array}{c}20.6407 \\
2\end{array}$ & 19.64 & $\begin{array}{c}1.95979 \\
1\end{array}$ & $\begin{array}{c}2.18610 \\
8\end{array}$ & $\begin{array}{c}3.99401 \\
6\end{array}$ & $\begin{array}{c}1.00071 \\
5\end{array}$ \\
\hline 10 & $\begin{array}{c}1.21998 \\
3\end{array}$ & 2.80978 & $\begin{array}{c}1.49748 \\
9\end{array}$ & $\begin{array}{c}3.02721 \\
6\end{array}$ & $\begin{array}{c}24.6900 \\
2\end{array}$ & $\begin{array}{c}23.1002 \\
2\end{array}$ & $\begin{array}{c}24.4125 \\
1\end{array}$ & $\begin{array}{c}22.8827 \\
8\end{array}$ & 25.91 & $\begin{array}{c}1.21998 \\
3\end{array}$ & 2.80978 & $\begin{array}{c}1.49748 \\
9\end{array}$ & $\begin{array}{c}3.02721 \\
6\end{array}$ \\
\hline 11 & $\begin{array}{c}2.72246 \\
6\end{array}$ & $\begin{array}{c}2.28721 \\
6\end{array}$ & 2.6361 & $\begin{array}{c}2.46289 \\
5\end{array}$ & $\begin{array}{c}193.677 \\
5\end{array}$ & $\begin{array}{c}194.112 \\
8\end{array}$ & $\begin{array}{c}193.763 \\
9\end{array}$ & $\begin{array}{c}193.937 \\
1\end{array}$ & 196.4 & $\begin{array}{c}2.72246 \\
6\end{array}$ & $\begin{array}{c}2.28721 \\
6\end{array}$ & 2.6361 & $\begin{array}{c}2.46289 \\
5\end{array}$ \\
\hline 12 & $\begin{array}{c}1.14023 \\
5\end{array}$ & $\begin{array}{c}1.00417 \\
6\end{array}$ & $\begin{array}{c}1.17337 \\
3\end{array}$ & 1.14708 & $\begin{array}{c}25.1697 \\
7\end{array}$ & $\begin{array}{c}25.3058 \\
2\end{array}$ & $\begin{array}{c}25.1366 \\
3\end{array}$ & $\begin{array}{c}25.1629 \\
2\end{array}$ & 26.31 & $\begin{array}{c}1.14023 \\
5\end{array}$ & $\begin{array}{c}1.00417 \\
6\end{array}$ & $\begin{array}{c}1.17337 \\
3\end{array}$ & 1.14708 \\
\hline$I^{\prime}$ & $\begin{array}{c}2.01067 \\
6\end{array}$ & $\begin{array}{c}4.79947 \\
1\end{array}$ & $\begin{array}{c}1.49790 \\
1\end{array}$ & $\begin{array}{c}4.31196 \\
8\end{array}$ & $\begin{array}{c}167.389 \\
3\end{array}$ & $\begin{array}{c}164.600 \\
5\end{array}$ & $\begin{array}{c}167.902 \\
1\end{array}$ & 165.088 & 169.4 & $\begin{array}{c}2.01067 \\
6\end{array}$ & $\begin{array}{c}4.79947 \\
1\end{array}$ & $\begin{array}{c}1.49790 \\
1\end{array}$ & $\begin{array}{c}4.31196 \\
8\end{array}$ \\
\hline $2^{\prime}$ & $\begin{array}{c}1.64289 \\
5\end{array}$ & $\begin{array}{c}1.55768 \\
6\end{array}$ & $\begin{array}{c}1.63033 \\
4\end{array}$ & $\begin{array}{c}1.69628 \\
6\end{array}$ & $\begin{array}{c}128.342 \\
9\end{array}$ & $\begin{array}{c}128.257 \\
7\end{array}$ & $\begin{array}{c}128.330 \\
3\end{array}$ & $\begin{array}{c}128.396 \\
3\end{array}$ & 126.7 & $\begin{array}{c}1.64289 \\
5\end{array}$ & $\begin{array}{c}1.55768 \\
6\end{array}$ & $\begin{array}{c}1.63033 \\
4\end{array}$ & $\begin{array}{c}1.69628 \\
6\end{array}$ \\
\hline $3^{\prime}$ & $\begin{array}{c}2.71894 \\
3\end{array}$ & $\begin{array}{c}3.69636 \\
4\end{array}$ & $\begin{array}{c}4.33761 \\
8\end{array}$ & 3.16188 & $\begin{array}{c}144.008 \\
9\end{array}$ & $\begin{array}{c}144.986 \\
4\end{array}$ & $\begin{array}{c}145.627 \\
6\end{array}$ & $\begin{array}{c}144.451 \\
9\end{array}$ & $\begin{array}{c}141.2 \\
9\end{array}$ & $\begin{array}{c}2.71894 \\
3\end{array}$ & $\begin{array}{c}3.69636 \\
4\end{array}$ & $\begin{array}{c}4.33761 \\
8\end{array}$ & 3.16188 \\
\hline $4^{\prime}$ & $\begin{array}{c}0.59369 \\
1\end{array}$ & $\begin{array}{c}0.64697 \\
4\end{array}$ & $\begin{array}{c}0.79211 \\
1\end{array}$ & $\begin{array}{c}0.62159 \\
5\end{array}$ & $\begin{array}{c}16.6736 \\
9\end{array}$ & $\begin{array}{c}16.7269 \\
7\end{array}$ & $\begin{array}{c}16.8721 \\
1\end{array}$ & 16.7016 & 16.08 & $\begin{array}{c}0.59369 \\
1\end{array}$ & $\begin{array}{c}0.64697 \\
4\end{array}$ & $\begin{array}{c}0.79211 \\
1\end{array}$ & $\begin{array}{c}0.62159 \\
5\end{array}$ \\
\hline $5^{\prime}$ & $\begin{array}{c}0.63224 \\
1\end{array}$ & $\begin{array}{c}0.64424 \\
6\end{array}$ & $\begin{array}{c}0.90634 \\
3\end{array}$ & $\begin{array}{c}1.11081 \\
6\end{array}$ & $\begin{array}{c}19.9477 \\
6\end{array}$ & $\begin{array}{c}19.9357 \\
5\end{array}$ & $\begin{array}{c}19.6736 \\
6\end{array}$ & $\begin{array}{c}19.4691 \\
8\end{array}$ & 20.58 & $\begin{array}{c}0.63224 \\
1\end{array}$ & $\begin{array}{c}0.64424 \\
6\end{array}$ & $\begin{array}{c}0.90634 \\
3\end{array}$ & $\begin{array}{c}1.11081 \\
6\end{array}$ \\
\hline & \multicolumn{4}{|c|}{$\begin{array}{c}\text { Isotropic Tensors } \\
\left({ }^{1} \mathrm{H}\right)\end{array}$} & \multicolumn{4}{|c|}{$\begin{array}{l}\text { Calculated Chemical } \\
\text { Shifts }\left({ }^{1} \mathrm{H}\right)^{\text {a }}\end{array}$} & & \multicolumn{4}{|c|}{$\left|\delta_{\text {teo }}-\delta_{\text {exp }}\right|$} \\
\hline Pos & $3 R, 4 R$ & $3 S, 4 R$ & $3 R, 4 S$ & $3 S, 4 S$ & $3 R, 4 R$ & $3 S, 4 R$ & $3 R, 4 S$ & $3 S, 4 S$ & Exp. & $3 R, 4 R$ & $3 S, 4 R$ & $3 R, 4 S$ & $3 S, 4 S$ \\
\hline 3 & $\begin{array}{c}0.24827 \\
8\end{array}$ & $\begin{array}{c}0.09657 \\
6\end{array}$ & $\begin{array}{c}0.03826 \\
7\end{array}$ & 0.24856 & $\begin{array}{c}4.11827 \\
8\end{array}$ & $\begin{array}{c}3.77342 \\
4\end{array}$ & $\begin{array}{c}3.90826 \\
7\end{array}$ & 4.11856 & 3.87 & $\begin{array}{c}0.24827 \\
8\end{array}$ & $\begin{array}{c}0.09657 \\
6\end{array}$ & $\begin{array}{c}0.03826 \\
7\end{array}$ & 0.24856 \\
\hline 4 & $\begin{array}{c}0.08965 \\
9\end{array}$ & $\begin{array}{c}0.13819 \\
6\end{array}$ & $\begin{array}{c}0.05101 \\
9\end{array}$ & $\begin{array}{c}0.05600 \\
9\end{array}$ & $\begin{array}{c}6.02965 \\
9\end{array}$ & $\begin{array}{c}5.80180 \\
4\end{array}$ & $\begin{array}{c}5.88898 \\
1\end{array}$ & $\begin{array}{c}5.88399 \\
1\end{array}$ & 5.94 & $\begin{array}{c}0.08965 \\
9\end{array}$ & $\begin{array}{c}0.13819 \\
6\end{array}$ & $\begin{array}{c}0.05101 \\
9\end{array}$ & $\begin{array}{c}0.05600 \\
9\end{array}$ \\
\hline 5 & $\begin{array}{c}0.14029 \\
1\end{array}$ & $\begin{array}{c}0.52535 \\
1\end{array}$ & $\begin{array}{c}0.05175 \\
6\end{array}$ & 0.24881 & $\begin{array}{c}8.01029 \\
1\end{array}$ & $\begin{array}{c}8.39535 \\
1\end{array}$ & $\begin{array}{c}7.92175 \\
6\end{array}$ & 8.11881 & 87 & $\begin{array}{c}0.14029 \\
1\end{array}$ & $\begin{array}{c}0.52535 \\
1\end{array}$ & $\begin{array}{c}0.05175 \\
6\end{array}$ & 0.24881 \\
\hline 7 & $\begin{array}{c}0.00929 \\
7\end{array}$ & $\begin{array}{c}0.04780 \\
6\end{array}$ & $\begin{array}{c}0.01213 \\
9\end{array}$ & 0.05665 & $\begin{array}{c}7.83929 \\
7\end{array}$ & $\begin{array}{c}7.87780 \\
6\end{array}$ & $\begin{array}{c}7.81786 \\
1\end{array}$ & 7.88665 & 7.83 & $\begin{array}{c}0.00929 \\
7\end{array}$ & $\begin{array}{c}0.04780 \\
6\end{array}$ & $\begin{array}{c}0.01213 \\
9\end{array}$ & 0.05665 \\
\hline 8 & $\begin{array}{c}0.07695 \\
9\end{array}$ & 0.0503 & $\begin{array}{c}0.11523 \\
2\end{array}$ & $\begin{array}{c}0.05790 \\
5\end{array}$ & $\begin{array}{c}6.80304 \\
1\end{array}$ & 6.8297 & $\begin{array}{c}6.76476 \\
8\end{array}$ & $\begin{array}{c}6.82209 \\
5\end{array}$ & 6.88 & $\begin{array}{c}0.07695 \\
9\end{array}$ & 0.0503 & $\begin{array}{c}0.11523 \\
2\end{array}$ & $\begin{array}{c}0.05790 \\
5\end{array}$ \\
\hline 9 & $\begin{array}{c}0.01404 \\
2\end{array}$ & $\begin{array}{c}0.07609 \\
7\end{array}$ & $\begin{array}{c}0.02592 \\
4\end{array}$ & $\begin{array}{c}0.01359 \\
1\end{array}$ & $\begin{array}{c}1.34404 \\
2\end{array}$ & $\begin{array}{c}1.40609 \\
7\end{array}$ & $\begin{array}{c}1.30407 \\
6\end{array}$ & $\begin{array}{c}1.31640 \\
9\end{array}$ & 1.33 & $\begin{array}{c}0.01404 \\
2\end{array}$ & $\begin{array}{c}0.07609 \\
7\end{array}$ & $\begin{array}{c}0.02592 \\
4\end{array}$ & $\begin{array}{c}0.01359 \\
1\end{array}$ \\
\hline 10 & $\begin{array}{c}0.01576 \\
4\end{array}$ & 0.02308 & $\begin{array}{c}0.00211 \\
3\end{array}$ & $\begin{array}{c}0.01298 \\
4\end{array}$ & $\begin{array}{c}1.48423 \\
6\end{array}$ & 1.52308 & $\begin{array}{c}1.49788 \\
7\end{array}$ & $\begin{array}{c}1.48701 \\
6\end{array}$ & 1.5 & $\begin{array}{c}0.01576 \\
4\end{array}$ & 0.02308 & $\begin{array}{c}0.00211 \\
3\end{array}$ & $\begin{array}{c}0.01298 \\
4\end{array}$ \\
\hline 12 & $\begin{array}{c}0.01827 \\
2\end{array}$ & $\begin{array}{c}0.00777 \\
8\end{array}$ & $\begin{array}{c}0.04584 \\
5\end{array}$ & $\begin{array}{c}0.00390 \\
8\end{array}$ & $\begin{array}{c}2.49172 \\
8\end{array}$ & $\begin{array}{c}2.50222 \\
2\end{array}$ & $\begin{array}{c}2.46415 \\
5\end{array}$ & $\begin{array}{c}2.51390 \\
8\end{array}$ & 2.51 & $\begin{array}{c}0.01827 \\
2\end{array}$ & $\begin{array}{c}0.00777 \\
8\end{array}$ & $\begin{array}{c}0.04584 \\
5\end{array}$ & $\begin{array}{c}0.00390 \\
8\end{array}$ \\
\hline $3^{\prime}$ & $\begin{array}{c}0.30793 \\
6\end{array}$ & $\begin{array}{c}0.32651 \\
1\end{array}$ & $\begin{array}{c}0.45787 \\
3\end{array}$ & 0.39248 & $\begin{array}{c}6.50793 \\
6\end{array}$ & $\begin{array}{c}6.52651 \\
1\end{array}$ & $\begin{array}{c}6.65787 \\
3\end{array}$ & 6.59248 & 6.2 & $\begin{array}{c}0.30793 \\
6\end{array}$ & $\begin{array}{c}0.32651 \\
1\end{array}$ & $\begin{array}{c}0.45787 \\
3\end{array}$ & 0.39248 \\
\hline $4^{\prime}$ & $\begin{array}{c}0.10151 \\
7\end{array}$ & 0.00757 & $\begin{array}{c}0.03165 \\
9\end{array}$ & $\begin{array}{c}0.01491 \\
6\end{array}$ & $\begin{array}{c}1.93848 \\
3\end{array}$ & 2.03243 & $\begin{array}{c}2.00834 \\
1\end{array}$ & $\begin{array}{c}2.05491 \\
6\end{array}$ & 2.04 & $\begin{array}{c}0.10151 \\
7\end{array}$ & 0.00757 & $\begin{array}{c}0.03165 \\
9\end{array}$ & $\begin{array}{c}0.01491 \\
6\end{array}$ \\
\hline $5^{\prime}$ & $\begin{array}{c}0.04879 \\
1\end{array}$ & 0.07491 & $\begin{array}{c}0.04155 \\
6\end{array}$ & 0.04062 & $\begin{array}{c}1.88120 \\
9\end{array}$ & 1.85509 & $\begin{array}{c}1.97155 \\
6\end{array}$ & 1.97062 & 1.93 & $\begin{array}{c}0.04879 \\
1\end{array}$ & 0.07491 & $\begin{array}{c}0.04155 \\
6\end{array}$ & 0.04062 \\
\hline & & & & & & & & & MAE & $\begin{array}{c}1.20537 \\
1\end{array}$ & $\begin{array}{c}1.55132 \\
5\end{array}$ & 1.1521 & $\begin{array}{c}1.64138 \\
6\end{array}$ \\
\hline & & & & & & & & & $\mathrm{R}^{2}$ & 0.9993 & 0.9991 & 0.9994 & 0.9989 \\
\hline
\end{tabular}


${ }^{a}$ Chemical shifts were derived from application of scaling factors (slope $=-1.0767$, intercept $\left.=31.9477\right)$ to the ${ }^{1} \mathrm{H}$ NMR shielding tensors and scaling factors (slope $=-1.0522$, intercept $=181.2412$ ) to the ${ }^{13} \mathrm{C}$ NMR shielding tensors computed at the B3LYP/6-311+G(2d,p)//M06-2X/6-31+G(d,p) level of theory.

Table S2. Calculated Coupling Constants (Fermi Contacts) at DFT M062X/6-31+g(d,p) Level of Theory for the Proposed 2,2-Dimethyl-3-Hidroxy-4-(1-Angeloyloxy)Chromane Structure.

\begin{tabular}{c|ccccccccc}
\multicolumn{1}{c}{ Calculated coupling constants (J in Hz) } \\
\hline $\mathrm{J}_{\text {pos }}$ & $3 R, 4 R$ & $3 S, 4 R$ & $3 R, 4 S$ & $3 S, 4 S$ & Exp. & $3 R, 4 R$ & $3 S, 4 R$ & $3 R, 4 S$ & $3 S, 4 S$ \\
$m-7,8$ & 0.128457 & 0.159424 & 0.095777 & 0.139514 & 1.90 & 2.02846 & 2.059424 & 1.995777 & 2.039514 \\
$o-5,7$ & 0.885118 & 0.873674 & 0.893049 & 0.891844 & 8.60 & 7.71488 & 7.726326 & 7.706951 & 7.708156 \\
3,4 & 2.642418 & 5.570622 & 0.181298 & 3.395483 & 7.56 & 4.91758 & 1.989378 & 7.741298 & 4.164517 \\
$3^{\prime}, 4^{\prime}$ & 0.335756 & 0.39737 & 0.363328 & 0.42743 & 7.30 & 6.96424 & 6.90263 & 6.936672 & 6.87257 \\
$3^{\prime}, 5^{\prime}$ & 0.318399 & 0.323625 & 0.318484 & 0.323624 & 1.50 & 1.81840 & 1.823625 & 1.818484 & 1.823624 \\
& & & & & MAE & 0.86203 & 1.464943 & 0.370387 & 1.035579
\end{tabular}


Table S3. Geometry Optimization and Boltzmann Distribution at M062X/6-31+g(d,p) Level of Theory for the Diastereomers of the Proposed 2,2-Dimethyl-3-Hidroxy-4-(1-Angeloyloxy)Chromane Structure.

\begin{tabular}{|c|c|c|c|}
\hline Conf & Gibbs free energy (hatree) & $\Delta G(\mathrm{kcal} / \mathrm{mol})$ & Population \\
\hline $3 R, 4 R \_1$ & -1074.593187 & 0 & 0.288996356 \\
\hline $3 R, 4 R \_2$ & -1074.592683 & 0.316264738 & 0.169354623 \\
\hline $3 R, 4 R \_3$ & -1074.592664 & 0.328187416 & 0.165976817 \\
\hline $3 R, 4 R \_4$ & -1074.592645 & 0.340110095 & 0.162666382 \\
\hline $3 R, 4 R \_5$ & -1074.592181 & 0.631274456 & 0.099454116 \\
\hline $3 R, 4 R \_6$ & -1074.592072 & 0.699672981 & 0.08859874 \\
\hline $3 R, 4 R \_7$ & -1074.590877 & 1.449546714 & 0.024952967 \\
\hline Conf & Gibbs free energy (hatree) & $\Delta \mathrm{G}(\mathrm{kcal} / \mathrm{mol})$ & Population \\
\hline $3 S, 4 R \_1$ & -1074.593386 & 0 & 0.505078137 \\
\hline $3 S, 4 R \_2$ & -1074.592831 & 0.348267717 & 0.280399623 \\
\hline $3 S, 4 R \_3$ & -1074.592008 & 0.864707953 & 0.117160312 \\
\hline $3 S, 4 R \_4$ & -1074.591384 & 1.256273819 & 0.060453914 \\
\hline $3 S, 4 R \_5$ & -1074.590661 & 1.709963115 & 0.028085242 \\
\hline $3 S, 4 R \_6$ & -1074.589569 & 2.39520338 & 0.008822771 \\
\hline Conf & Gibbs free energy (hatree) & $\Delta \mathrm{G}(\mathrm{kcal} / \mathrm{mol})$ & Population \\
\hline $3 R, 4 S_{-} 1$ & -1074.594048 & 0 & 0.247207702 \\
\hline $3 R, 4 S \_2$ & -1074.593862 & 0.116716748 & 0.202958748 \\
\hline $3 R, 4 S \_3$ & -1074.593403 & 0.404743563 & 0.12474848 \\
\hline $3 R, 4 S \_4$ & -1074.593294 & 0.473142088 & 0.111132234 \\
\hline $3 R, 4 S \_5$ & -1074.593282 & 0.4806722 & 0.109727117 \\
\hline $3 R, 4 S \_6$ & -1074.593253 & 0.498869973 & 0.106404324 \\
\hline $3 R, 4 S \_7$ & -1074.59316 & 0.557228347 & 0.096412238 \\
\hline $3 R, 4 S \_8$ & -1074.58825 & 3.638299501 & 0.0005285 \\
\hline Conf & Gibbs free energy (hatree) & $\Delta \mathrm{G}(\mathrm{kcal} / \mathrm{mol})$ & Population \\
\hline $3 S, 4 S_{-} 1$ & -1074.59327 & 0 & 0.305934493 \\
\hline $3 S, 4 S \_2$ & -1074.593058 & 0.133031993 & 0.244343647 \\
\hline $3 S, 4 S \_3$ & -1074.593013 & 0.161269916 & 0.232958337 \\
\hline $3 S, 4 S \_4$ & -1074.592944 & 0.204568064 & 0.216522635 \\
\hline $3 S, 4 S \_5$ & -1074.58653 & 4.229413356 & 0.000240887 \\
\hline
\end{tabular}


Table S4. DP4+ Analysis of the Proposed 2,2-Dimethyl-3-Hidroxy-4-(1-Angeloyloxy)Chromane

\section{Structure.}

\begin{tabular}{|c|c|c|c|c|c|c|c|c|}
\hline 1 & \multirow{2}{*}{\multicolumn{2}{|c|}{$\begin{array}{l}\text { Functional } \\
\text { B3LYP }\end{array}$}} & \multicolumn{2}{|c|}{ Solvent? } & \multirow{2}{*}{\multicolumn{2}{|c|}{$\begin{array}{l}\text { Basis Set } \\
6-311+G(d, p)\end{array}$}} & \multicolumn{2}{|c|}{ Type of Data } \\
\hline 2 & & & \multicolumn{2}{|c|}{ PCM } & & & \multicolumn{2}{|c|}{ Shielding Tensors } \\
\hline \multicolumn{9}{|c|}{3} \\
\hline 12 & & & DP4+ & $5.58 \%$ & $0.00 \%$ & - $94.42 \%$ & $0.00 \%$ & - \\
\hline 14 & Nuclei & sp2? & Experimental & Isomer 1 & Isomer 2 & Isomer 3 & Isomer 4 & Isomer 5 \\
\hline 15 & C & $\mathrm{x}$ & 130.4 & 47.5583044 & 47.1666134 & 47.9645081 & 47.3677227 & \\
\hline 16 & C & $\mathrm{x}$ & 129.72 & 43.2682851 & 41.329407 & 46.3401528 & 46.2653961 & \\
\hline 17 & C & $\mathrm{x}$ & 119.53 & 55.6909094 & 56.489153 & 55.6647983 & 57.9951408 & \\
\hline 18 & C & $\mathrm{x}$ & 157.2 & 16.9692383 & 17.5621785 & 17.6740514 & 17.8968607 & \\
\hline 19 & C & $\mathrm{x}$ & 117.46 & 60.0299503 & 60.1487952 & 60.8397961 & 60.6538224 & \\
\hline 20 & C & $\mathrm{x}$ & 130.4 & 45.4029734 & 46.3182009 & 46.6412862 & 46.4812181 & \\
\hline 21 & C & $\mathrm{x}$ & 196.4 & -22.5463018 & -23.0042708 & -22.6371753 & -22.8194217 & \\
\hline 22 & C & & 26.31 & 154.757573 & 154.614412 & 154.792441 & 154.764776 & \\
\hline 23 & C & & 71.86 & 111.900408 & 109.920984 & 105.676848 & 110.179704 & \\
\hline 24 & C & & 74.19 & 104.343081 & 106.687945 & 103.860004 & 112.382834 & \\
\hline 25 & C & & 79.63 & 100.160809 & 100.808435 & 98.1838066 & 98.186124 & \\
\hline 26 & C & & 19.64 & 162.638084 & 158.275769 & 164.778496 & 159.52304 & \\
\hline 27 & C & & 25.91 & 155.262364 & 156.935149 & 155.554356 & 157.163935 & \\
\hline 28 & C & $\mathrm{x}$ & 169.4 & 5.11415363 & 8.04852333 & 4.57461139 & 7.53557299 & \\
\hline 29 & C & $\mathrm{x}$ & 126.7 & 46.1988057 & 46.2884626 & 46.212023 & 46.1426278 & \\
\hline 30 & C & $x$ & 141.29 & 29.7149899 & 28.6865477 & 28.0118199 & 29.2489316 & \\
\hline 31 & C & & 16.08 & 163.697143 & 163.641078 & 163.488365 & 163.667782 & \\
\hline 32 & C & & 20.58 & 160.252168 & 160.264799 & 160.540578 & 160.755725 & \\
\hline \multicolumn{9}{|l|}{33} \\
\hline \multicolumn{9}{|l|}{34} \\
\hline \multicolumn{9}{|l|}{35} \\
\hline 36 & H & $\mathrm{x}$ & 7.87 & 23.3230201 & 22.9084256 & 23.4183448 & 23.2061771 & \\
\hline 37 & H & $\mathrm{x}$ & 6.88 & 24.6228657 & 24.5941625 & 24.6640744 & 24.6023502 & \\
\hline 38 & H & $x$ & 7.83 & 23.5071291 & 23.4656661 & 23.5302095 & 23.4561434 & \\
\hline 39 & H & & 2.51 & 29.264856 & 29.253558 & 29.2945444 & 29.2409748 & \\
\hline 40 & H & & 5.94 & 25.4555663 & 25.7008973 & 25.6070337 & 25.6124064 & \\
\hline 41 & H & & 3.87 & 27.51355 & 27.8848544 & 27.7396688 & 27.5132463 & \\
\hline 42 & H & & 1.33 & 30.5005704 & 30.4337556 & 30.5436011 & 30.5303223 & \\
\hline 43 & H & & 1.5 & 30.349623 & 30.3078 & 30.334925 & 30.3466295 & \\
\hline 44 & H & $\mathrm{x}$ & 6.2 & 24.9406048 & 24.9206054 & 24.7791684 & 24.8495772 & \\
\hline 45 & H & & 2.04 & 29.8605349 & 29.7593829 & 29.785319 & 29.7351716 & \\
\hline 46 & H & & 1.93 & 29.9222024 & 29.9503241 & 29.8249257 & 29.8259329 & \\
\hline 47 & & & & & & & & \\
\hline
\end{tabular}




\section{Table S5. J-DP4 Analysis of the Proposed 2,2-Dimethyl-3-Hidroxy-4-(1-Angeloyloxy)Chromane}

\section{Structure.}

\begin{tabular}{|c|c|c|c|c|c|c|c|}
\hline 8 & \multicolumn{2}{|c|}{ Isomer $\mathrm{N} N$ - } & 1 & \multirow{2}{*}{$\frac{2}{1.54}$} & \multirow{2}{*}{$\frac{3}{59.54}$} & \multirow{2}{*}{$\frac{4}{10.37}$} & \multirow{2}{*}{5} \\
\hline 9 & \multirow{5}{*}{ DP4 (\%) } & H & 28.55 & & & & \\
\hline 10 & & C & 34.73 & 0.15 & 65.10 & 0.01 & - \\
\hline 11 & & $\mathrm{H}+\mathrm{C}$ & 20.37 & 0.00 & 79.63 & 0.00 & - \\
\hline 12 & & 1 & 15.12 & 1.81 & 75.00 & 8.07 & - \\
\hline 13 & & all data & 4.90 & 0.00 & 95.10 & 0.00 & - \\
\hline 14 & Type & Exp & 1 & 2 & 3 & 4 & 5 \\
\hline 15 & C & 130.4 & 127.051 & 127.4231 & 126.665 & 127.232 & \\
\hline 16 & C & 129.72 & 131.128 & 132.9707 & 128.209 & 128.2796 & \\
\hline 17 & C & 119.53 & 119.322 & 118.5631 & 119.347 & 117.1318 & \\
\hline 18 & C & 157.2 & 156.122 & 155.5588 & 155.453 & 155.2408 & \\
\hline 19 & C & 117.46 & 115.198 & 115.085 & 114.428 & 114.605 & \\
\hline 20 & C & 130.4 & 129.099 & 128.2294 & 127.922 & 128.0745 & \\
\hline 21 & C & 196.4 & 193.678 & 194.1128 & 193.764 & 193.9371 & \\
\hline 22 & C & 26.31 & 25.1698 & 25.30582 & 25.1366 & 25.16292 & \\
\hline 23 & C & 71.86 & 65.9008 & 67.782 & 71.8156 & 67.53611 & \\
\hline 24 & C & 74.19 & 73.0832 & 70.85464 & 73.5423 & 65.44228 & \\
\hline 25 & c & 79.63 & 77.058 & 76.44247 & 78.9369 & 78.93469 & \\
\hline 26 & C & 19.64 & 17.6802 & 21.82611 & 15.646 & 20.64072 & \\
\hline 27 & c & 25.91 & 24.69 & 23.10022 & 24.4125 & 22.88278 & \\
\hline 28 & c & 169.4 & 167.389 & 164.6005 & 167.902 & 165.088 & \\
\hline 29 & C & 126.7 & 128.343 & 128.2577 & 128.33 & 128.3963 & \\
\hline 30 & C & 141.29 & 144.009 & 144.9864 & 145.628 & 144.4519 & \\
\hline 31 & C & 16.08 & 16.6737 & 16.72697 & 16.8721 & 16.7016 & \\
\hline 32 & C & 20.58 & 19.9478 & 19.93575 & 19.6737 & 19.46918 & \\
\hline \multicolumn{8}{|l|}{33} \\
\hline \multicolumn{8}{|l|}{34} \\
\hline \multicolumn{8}{|l|}{35} \\
\hline 36 & H & 7.87 & 8.01029 & 8.395351 & 7.92176 & 8.11881 & \\
\hline 37 & $\mathrm{H}$ & 6.88 & 6.80304 & 6.8297 & 6.76477 & 6.822095 & \\
\hline 38 & H & 7.83 & 7.8393 & 7.877806 & 7.81786 & 7.88665 & \\
\hline 39 & H & 2.51 & 2.49173 & 2.502222 & 2.46415 & 2.513908 & \\
\hline 40 & H & 5.94 & 6.02966 & 5.801804 & 5.88898 & 5.883991 & \\
\hline 41 & H & 3.87 & 4.11828 & 3.773424 & 3.90827 & 4.11856 & \\
\hline 42 & H & 1.33 & 1.34404 & 1.406097 & 1.30408 & 1.316409 & \\
\hline 43 & H & 1.5 & 1.48424 & 1.52308 & 1.49789 & 1.487016 & \\
\hline 44 & H & 6.2 & 6.50794 & 6.526511 & 6.65787 & 6.59248 & \\
\hline 45 & $\mathrm{H}$ & 2.04 & 1.93848 & 2.03243 & 2.00834 & 2.054916 & \\
\hline 46 & H & 1.93 & 1.88121 & 1.85509 & 1.97156 & 1.97062 & \\
\hline \multicolumn{8}{|l|}{47} \\
\hline \multicolumn{8}{|l|}{48} \\
\hline \multicolumn{8}{|l|}{49} \\
\hline 50 & J & 1.90 & 2.02846 & 2.059424 & 1.99578 & 2.039514 & \\
\hline 51 & J & 8.60 & 7.71488 & 7.726326 & 7.70695 & 7.708156 & \\
\hline 52 & J & 7.30 & 6.96424 & 6.90263 & 6.93667 & 6.87257 & \\
\hline 53 & J & 1.50 & 1.81840 & 1.823625 & 1.81848 & 1.823624 & \\
\hline 54 & J & 7.56 & 4.91758 & 1.989378 & 7.7413 & 4.164517 & \\
\hline & 4 & Main & Unscale & Sca & Err & ors & \\
\hline
\end{tabular}

